hep-th/9906055

CALT-68-2228

DAMTP-1999-74

\title{
Dualities of Type 0 Strings
}

\author{
Oren Bergman' \\ California Institute of Technology \\ Pasadena, CA 91125, USA \\ Matthias R. Gaberdiell \\ Department of Applied Mathematics and Theoretical Physics \\ University of Cambridge, Silver Street, \\ Cambridge CB3 9EW, U.K.
}

June 1999

\begin{abstract}
It is conjectured that the two closed bosonic string theories, Type 0A and Type 0B, correspond to certain supersymmetry breaking orbifold compactifications of M-theory. Various implications of this conjecture are discussed, in particular the behaviour of the tachyon at strong coupling and the existence of non-perturbative fermionic states in Type 0A. The latter are shown to correspond to bound states of Type 0A D-particles, thus providing further evidence for the conjecture. We also give a comprehensive description of the various Type 0 closed and open string theories.
\end{abstract}

PACS 11.25.-w, 11.25.Sq

${ }^{*}$ E-mail address: bergman@theory.caltech.edu

${ }^{\dagger}$ E-mail address: M.R.Gaberdiel@damtp.cam.ac.uk 


\section{Introduction}

Closed string theories are highly constrained by their intrinsic consistency conditions, in particular modular invariance, and only finitely many uncompactified theories are known to exist. Other than the four supersymmetric theories, there exist nine non-supersymmetric closed string theories in ten dimensions: the bosonic Type 0A and Type 0B theories, as well as seven nonsupersymmetric heterotic string theories [1]. The first two are obtained by projecting Type IIA and Type IIB, respectively, by the discrete symmetry generated by the operator $(-1)^{F^{s}}$, where $F^{s}$ denotes the spacetime fermion number; this acts as \pm 1 on spacetime bosons and fermions, respectively, and is therefore equivalent to a rotation by $2 \pi$. The non-supersymmetric heterotic theories correspond to projections of the two supersymmetric heterotic theories by $(-1)^{F^{s}} \cdot Y$, where $Y$ is an automorphism of the internal weight lattice. The resulting theories are uniquely characterised by their gauge groups, which are $S O(32), E_{8} \times S O(16), S O(24) \times S O(8),\left(E_{7} \times S U(2)\right)^{2}$, $U(16), E_{8}$, and $S O(16) \times S O(16)$. All of the above theories, except for the $S O(16) \times S O(16)$ string, have tachyons. This has usually been taken to imply either a perturbative inconsistency, or an instability of the given background. The latter requires higher order terms in the tachyon potential to stabilize its vacuum expectation value.

Type 0B string theory, in particular, has attracted recent attention as a non-supersymmetric setting for a gauge theory - (super)gravity correspondence [2, 3]. This theory possesses Dirichlet 3-branes, whose world-volume field theory is a non-supersymmetric four-dimensional gauge theory. In the limit of a large number of D3-branes, a variant of the Maldacena conjecture [4] suggests a correspondence between the gauge theory on the brane and Type 0B gravity in a certain background. This has led to novel insights into the nature of these non-supersymmetric gauge theories. An interesting feature of the limit is that the tachyon, due to its coupling to the $\mathrm{R}-\mathrm{R}$ fields, actually becomes massive.

In contrast to closed string theories, the consistency conditions for open string theories are not completely understood. The only condition that is normally imposed is the absence of massless tadpoles in the closed string channel of the annulus amplitude [5]. This has led, in the supersymmetric case, to a unique open string theory with gauge group $S O(32)$, namely Type I. In the non-supersymmetric case there exist a large number of tadpole-free theories, which can be obtained as world-sheet parity (orientifold) projections of Type 0A and Type 0B [6, 0, 8, 9]; these will be reviewed briefly in section 2.3 , where we explain their microscopic realisation in terms of D-branes. It is not known, however, whether these are the only open string theories in ten dimensions. Like their closed string ancestors, these theories contain D-branes, and may therefore be relevant to the study of non-supersymmetric gauge theories [9].

There exists by now a convincing picture of a web of string dualities that connect the various supersymmetric string theories (for a review see for example [10]). All evidence points towards an underlying eleven-dimensional structure, known as M-theory, from which the five ten-dimensional

\footnotetext{
${ }^{1}$ We shall not discuss the $D=26$ bosonic string in this paper.
} 
superstring theories emerge in certain limits. In particular, Type IIA string theory and Heterotic string theory with gauge group $E_{8} \times E_{8}$ correspond to compactification on a circle and an interval, respectively, whereas Type IIB and both $S O(32)$ string theories correspond to the zero volume limit of compactification on a torus and a cylinder, respectively. It is natural to ask whether the non-supersymmetric strings are also part of the web of dualities, and whether they can be described as certain limits of M-theory as well. Early studies indicate that the different nonsupersymmetric string theories may indeed be related by dualities [7, 11], but the question of tachyons has so far not been addressed.

In this paper we argue that Type 0A and Type 0B string theories can indeed be described as certain supersymmetry breaking compactifications of M-theory. More specifically, Type 0A corresponds to M-theory on $\mathbf{S}^{1} /(-1)^{F^{s}} \cdot S$, where $S$ denotes a half-shift along the circle, and Type 0B corresponds to the zero volume limit of M-theory on $\mathbf{T}^{2} /(-1)^{F^{s}} \cdot S$, where the shift $S$ is along one of the cycles of the torus. In analogy with Type IIA and Type IIB, the string coupling constant is given by the radius of the circle in the first case, and by the imaginary part of the complex structure of the torus in the second case. As in the case of Type IIB, this realisation of Type $0 \mathrm{~B}$ suggests that the theory possesses an $S L(2, \mathbb{Z})$ symmetry.

If true, our conjecture implies that the Type 0A tachyon becomes massive at strong coupling, and that the theory flows dynamically to infinite coupling, i.e. that the eleventh dimension decompactifies completely. Similarly, the tachyon of Type 0B in nine dimensions becomes massive for a sufficiently large value of $g^{(0 B)} / R^{(0 B)}$. At large coupling and fixed radius the theory flows then to weakly coupled ten-dimensional Type IIA, whereas at fixed coupling and small radius it flows to eleven-dimensional M-theory.

The relation to M-theory also implies that the Type 0 theories must contain non-perturbative fermionic states, even though their perturbative spectrum is purely bosonic. An independent confirmation of the existence of such states therefore serves as evidence for the conjecture. We shall argue below that such states do indeed exist in Type 0A, and that they correspond to certain bound states of D-particles.

Finally, some of the open string models obtained as world-sheet parity projections of Type 0A and Type 0B are actually ruled out as a consequence of the relation to M-theory. For example, the orientifold of Type $0 \mathrm{~A}$ is not well-defined at finite coupling, since world-sheet parity cannot be lifted to a symmetry of the M-theory orbifold.

The paper is organised as follows. In section 2, we describe the main features of Type 0 strings, including the perturbative spectrum, D-branes, and the open string theories that are obtained by world-sheet parity projection. In section 3 we motivate and state our conjectures for Type 0A and Type 0B, and discuss their implications for the tachyons. In section 4 we describe the non-perturbative fermionic states of Type 0A. In section 5 we discuss the implications for the open string models, and in section 6 we present our conclusions and open questions. 


\section{Type 0 Strings}

Let us begin by describing the features of Type 0A and Type 0B in some detail. We shall work in the NS-R formalism, as this is more appropriate for theories without spacetime supersymmetry.

\subsection{Perturbative spectrum}

The spectra of the Type II theories is given by

$$
\begin{array}{ll}
\text { IIA : } & (N S+, N S+) \oplus(R+, R-) \oplus(N S+, R-) \oplus(R+, N S+) \\
\text { IIB : } & (N S+, N S+) \oplus(R+, R+) \oplus(N S+, R+) \oplus(R+, N S+),
\end{array}
$$

where the signs refer to the eigenvalues of $(-1)^{F_{L}}$ and $(-1)^{F_{R}}$, respectively. The effect of $(-1)^{F^{s}}$ in the untwisted sector is to retain the bosons (i.e. the states in the NS-NS and R-R sectors) and to remove the fermions (i.e. the states in the NS-R and R-NS sectors). In the two remaining sectors, the GSO projection acts in the usual way

$$
\begin{array}{ll}
\text { NS-NS: } & P_{G S O, U}=\frac{1}{4}\left(1+(-1)^{F_{L}}\right)\left(1+(-1)^{F_{R}}\right) \\
\text { R-R: } & P_{G S O, U}=\frac{1}{4}\left(1+(-1)^{F_{L}}\right)\left(1 \pm(-1)^{F_{R}}\right),
\end{array}
$$

where the + sign corresponds to Type IIB, and the - sign to Type IIA. In the twisted sector, the effect of $(-1)^{F^{s}}$ is to reverse the GSO projection for both left and right-moving sectors. In addition only the states invariant under $(-1)^{F^{s}}$ (i.e. the bosons) are retained. Thus the states in the twisted sector are again in the NS-NS and the R-R sector, but their GSO projection is now

$$
\begin{array}{ll}
\text { NS-NS: } & P_{G S O, T}=\frac{1}{4}\left(1-(-1)^{F_{L}}\right)\left(1-(-1)^{F_{R}}\right) \\
\text { R-R: } & P_{G S O, T}=\frac{1}{4}\left(1-(-1)^{F_{L}}\right)\left(1 \mp(-1)^{F_{R}}\right),
\end{array}
$$

where now the - sign corresponds to Type IIB, and the + sign to Type IIA. Taking (2.2) and (2.3) together, we can describe the spectrum of Type 0A and Type 0B more compactly as the subspaces of the NS-NS and R-R sectors that are invariant under the GSO-projection

$$
\begin{array}{ll}
\text { NS-NS: } & P_{G S O}=\frac{1}{2}\left(1+(-1)^{F_{L}+F_{R}}\right) \\
\text { R-R: } & P_{G S O}=\frac{1}{2}\left(1 \pm(-1)^{F_{L}+F_{R}}\right) .
\end{array}
$$

The resulting spectrum is given by

$$
\begin{array}{ll}
\text { OA : } & (N S+, N S+) \oplus(N S-, N S-) \oplus(R+, R-) \oplus(R-, R+) \\
\text { 0B : } & (N S+, N S+) \oplus(N S-, N S-) \oplus(R+, R+) \oplus(R-, R-) .
\end{array}
$$


The NS-NS sector is the same for the two theories. In particular, the low lying states consist of the ground state tachyon (that is invariant under (2.4) since it is invariant under (2.3)), and the bosonic part of the supergravity multiplet, i.e. the graviton, Kalb-Ramond 2-form, and dilaton. On the other hand, the R-R sector is different for the two theories (as is familiar from Type IIA and Type IIB). There are no tachyonic states, and the massless states transform as

$$
\begin{array}{ll}
\text { 0A : } & \left(\mathbf{8}_{\mathbf{s}} \otimes \mathbf{8}_{\mathrm{c}}\right) \oplus\left(\mathbf{8}_{\mathbf{c}} \otimes \mathbf{8}_{\mathrm{s}}\right)=2 \cdot \mathbf{8}_{\mathbf{v}}+2 \cdot \mathbf{5 6} \\
\text { 0B : } & \left(\mathbf{8}_{\mathrm{s}} \otimes \mathbf{8}_{\mathrm{s}}\right) \oplus\left(\mathbf{8}_{\mathbf{c}} \otimes \mathbf{8}_{\mathbf{c}}\right)=2 \cdot \mathbf{1}+2 \cdot \mathbf{2 8}+\mathbf{7 0}
\end{array}
$$

In the case of Type 0A, the theory has two 1-forms and two 3-forms in the R-R sector, whereas Type 0B has two scalars, two 2-forms, and a 4-form (with an unrestricted 5-form field strength). The states in the R-R sector of Type 0A and Type 0B are therefore doubled compared to those in Type IIA and Type IIB.

\subsection{D-branes}

The doubling of the R-R tensor fields suggests that the D-brane spectrum of Type 0A and Type OB is doubled compared to that of the corresponding Type II theories. This is indeed confirmed by a careful analysis of the boundary states in the two theories [7]. Let us briefly summarise and clarify this analysis.

A typical boundary state in a world-sheet supersymmetric closed string theory is labelled by an integer $p \in\{-1, \ldots, 9\}$ that determines the number of Dirichlet and Neumann conditions, and two spin structures, $\eta$ and $\epsilon$ [5], where $\eta$ is either NS-NS or R-R and describes the closed string sector to which the state belongs, and $\epsilon= \pm$. The defining equations are

$$
\begin{aligned}
& \left.\begin{array}{rl}
\left(\alpha_{n}^{\mu}-\widetilde{\alpha}_{-n}^{\mu}\right)|B p, \epsilon\rangle_{\eta} & =0 \\
\left(\psi_{r}^{\mu}-i \epsilon \widetilde{\psi}_{-r}^{\mu}\right)|B p, \epsilon\rangle_{\eta} & =0
\end{array}\right\} \mu=0, \ldots, p \\
& \left.\begin{array}{rl}
\left(\alpha_{n}^{\mu}+\widetilde{\alpha}_{-n}^{\mu}\right)|B p, \epsilon\rangle_{\eta} & =0 \\
\left(\psi_{r}^{\mu}+i \epsilon \widetilde{\psi}_{-r}^{\mu}\right)|B p, \epsilon\rangle_{\eta} & =0
\end{array}\right\} \quad \mu=p+1, \ldots, 9 .
\end{aligned}
$$

Here $n \in \mathbb{Z}$, and $r \in \mathbb{Z}(\mathbb{Z}+1 / 2)$ for $\eta=\mathrm{R}$-R (NS-NS). Up to normalisation, these equations determine a unique state for each $\eta$ and $\epsilon$,

$$
|B p,+\rangle_{\mathrm{NS}-\mathrm{NS}}, \quad|B p,-\rangle_{\mathrm{NS}-\mathrm{NS}}, \quad|B p,+\rangle_{\mathrm{R}-\mathrm{R}}, \quad|B p,-\rangle_{\mathrm{R}-\mathrm{R}} .
$$

The boundary states must be physical closed string states, and thus must be invariant under the gauge symmetries of the closed string theory. These include the GSO transformation(s), as

\footnotetext{
${ }^{2}$ There are also equations for ghost and superghost oscillators, which are independent of $p$ and the spin structures.
} 
well as orientifold and orbifold transformations, where appropriate. For the Type II theories this implies that only the combinations

$$
\begin{array}{ll}
|B p,+\rangle_{\mathrm{NS}-\mathrm{NS}}+|B p,-\rangle_{\mathrm{NS}-\mathrm{NS}} & \text { for all } p \\
|B p,+\rangle_{\mathrm{R}-\mathrm{R}}+|B p,-\rangle_{\mathrm{R}-\mathrm{R}} & \text { for } p=\left\{\begin{array}{cc}
\text { even } & \text { IIA } \\
\text { odd } & \text { IIB }
\end{array}\right.
\end{array}
$$

are allowed." In the Type 0 theories on the other hand, all four boundary states in (2.8) are invariant under the GSO projection (2.4), with an analogous restriction on the value of $p$ in the R-R sector.

The key property of boundary states is that the tree-level amplitude that describes the propagation from one of them to another can be reexpressed, through world-sheet duality, as a one-loop open string vacuum amplitude. The relevant amplitudes are given by

$$
\begin{aligned}
\int d l\left\langle B p, \epsilon\left|e^{-l H_{\text {closed }}}\right| B p, \epsilon\right\rangle_{\mathrm{NS}-\mathrm{NS}} & =\frac{1}{2} \int \frac{d t}{t} \operatorname{Tr}_{N S}\left[e^{-t H_{\text {open }}}\right] \\
\int d l\left\langle B p, \epsilon\left|e^{-l H_{\text {closed }}}\right| B p,-\epsilon\right\rangle_{\mathrm{NS}-\mathrm{NS}} & =-\frac{1}{2} \int \frac{d t}{t} \operatorname{Tr}_{R}\left[e^{-t H_{\text {open }}}\right] \\
\int d l\left\langle B p, \epsilon\left|e^{-l H_{\text {closed }}}\right| B p, \epsilon\right\rangle_{\mathrm{R}-\mathrm{R}} & =\frac{1}{2} \int \frac{d t}{t} \operatorname{Tr}_{N S}\left[e^{-t H_{\text {open }}}(-1)^{F}\right] \\
\int d l\left\langle B p, \epsilon\left|e^{-l H_{\text {closed }}}\right| B p,-\epsilon\right\rangle_{\mathrm{R}-\mathrm{R}} & =-\frac{1}{2} \int \frac{d t}{t} \operatorname{Tr}_{R}\left[e^{-t H_{\text {open }}}(-1)^{F}\right]=0 .
\end{aligned}
$$

This leads to additional consistency conditions, since the spectrum of the open strings that are introduced by the presence of the boundary states, as well as their interactions with the closed strings, must be consistent. Typically, the D-brane states are therefore linear combinations of the boundary states; these have to satisfy the following two conditions:

(a) For any pair of D-branes, the open string amplitude corresponds to a partition function of an open string theory, i.e. it takes the form of a sum of traces over sets of open string states of the time evolution operator $e^{-t H_{\text {open }}}$.

(b) The open-closed vertex that describes the joining of the two ends of an open string to form a closed string is well-defined on physical states.

One is usually also interested in D-branes that are stable; this is equivalent to the condition that the spectrum of open strings that begin and end on the same D-brane is free of tachyons. If the underlying theory is supersymmetric, one may also want to impose the condition that the D-branes preserve some of the supersymmetry, i.e. that they are BPS saturated; this requires that the spectrum of open strings beginning and ending on the D-brane is supersymmetric.

\footnotetext{
${ }^{3}$ The normalisation of the state $|B p,-\rangle_{\text {NS-NS }}$ has been chosen to be minus that used in [5, 7 .
} 
For the Type II theories the BPS and stability conditions are equivalent. Th These select the linear combinations

$$
\text { Type II : } \quad|D p\rangle=\left(|B p,+\rangle_{\mathrm{NS}-\mathrm{NS}}+|B p,-\rangle_{\mathrm{NS}-\mathrm{NS}}\right) \pm\left(|B p,+\rangle_{\mathrm{R}-\mathrm{R}}+|B p,-\rangle_{\mathrm{R}-\mathrm{R}}\right),
$$

where $p$ is even (odd) for Type IIA (IIB). The relative sign of the R-R and NS-NS components determines the sign of the R-R charge carried by the D-brane, and therefore differentiates a brane from an anti-brane. By applying (2.10), it immediately follows that (a) is satisfied. It is also generally believed that (b) is satisfied for these states, although this has never been completely verified to our knowledge.

The NS-NS component of (2.11) actually satisfies (a) (and presumably (b)) by itself, but is non-supersymmetric, and in fact unstable due to the presence of an open string tachyon. The combinations given by

$$
|\widehat{D p}\rangle=\left(|B p,+\rangle_{\mathrm{NS}-\mathrm{NS}}+|B p,-\rangle_{\mathrm{NS}-\mathrm{NS}}\right)
$$

therefore correspond to unstable (and non-BPS) D-branes. As such, they are not really seen as part of the spectrum of Type II string theory. Nevertheless, unstable D-branes are very useful as intermediaries in describing descent relations among D-branes in the Type II theories [16, 17].

For the Type 0 theories, property (a) again implies that one should take either a combination of an NS-NS state and an R-R state, or just an NS-NS state, but again only the former will be stable. As for Type II theories, property (b) has not been completely analysed. However, one immediate implication of $(\mathbf{b})$ is that the spectrum of open strings that begin and end on the same D-brane must be free of fermions; if this were not so, closed string fermions, which are absent in the closed string spectrum, would emerge from the open-closed vertex. This condition implies (see [7] for a detailed discussion) that of the various (stable) states that are consistent with (a), only eight linear combinations remain

$$
|B p, \epsilon\rangle_{\mathrm{NS}-\mathrm{NS}} \pm\left|B p, \epsilon^{\prime}\right\rangle_{\mathrm{R}-\mathrm{R}}
$$

where $p$ is even (odd) in Type 0A (0B).

The number is further reduced by imposing condition (a) on the amplitudes involving two different D-branes. Th This requires that, out of the eight combinations above, we keep only the four with $\epsilon=\epsilon^{\prime}$, or only the four with $\epsilon=-\epsilon^{\prime}$. This is because the amplitude for one of the former states to propagate into one of the latter states corresponds to a trace over open string states with an insertion of $(-1)^{F}$, rather than 1 or a projection operator, and therefore violates condition (a). On the other hand, amplitudes involving only the states with $\epsilon=\epsilon^{\prime}$ (or $\left.\epsilon=-\epsilon^{\prime}\right)$

\footnotetext{
${ }^{4}$ In other theories, such as Type I, and certain orbifolds of Type II, a tachyon-free open string spectrum can also be achieved without supersymmetry, resulting in stable non-BPS D-branes [12, 13, 14, 15, 16.

${ }^{5}$ This condition was not considered in [7]; however all D-branes that were relevant for the description of the duality in that paper satisfied it. See also [13] for a related discussion in a different context.
} 
will correspond to traces over projected sets of open string states, and will therefore satisfy (a). Let us fix our conventions by keeping the four combinations with $\epsilon=\epsilon^{\prime}$; these describe the two possible D-branes (and D-antibranes),

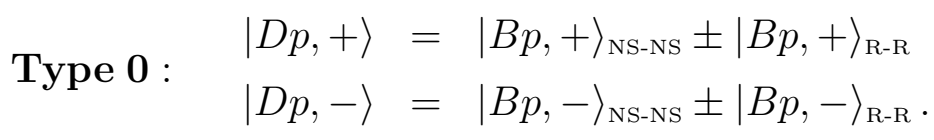

The above restriction was also obtained in [2] using a different method. There it was argued that D-branes that carry opposite charges with respect to the R-R fields in the twisted sector should also couple to the tachyon in the twisted NS-NS sector with opposite signs. The signs of these charges are in turn related to the spin structures as follows; the two R-R boundary states with $\epsilon^{\prime}= \pm$ carry equal (unit) charges under the untwisted R-R fields, and opposite charges under the twisted R-R fields. (This is an obvious consequence of the fact that Type II D-branes (2.11) are charged only under the untwisted fields.) Similarly, the two NS-NS boundary states couple to the tachyon with opposite signs. The argument of [2] then implies that the two spin structures must be correlated, reducing the allowed D-brane states to those in (2.14).

The open string spectra associated with these D-branes can easily be read off from the two relevant amplitudes,

$$
\begin{aligned}
\int d l\left\langle D p, \epsilon\left|e^{-l H_{\text {closed }}}\right| D p, \epsilon\right\rangle & =\int \frac{d t}{t} \operatorname{Tr}_{N S}\left[e^{-t H_{\text {open }}} \frac{1}{2}\left(1+(-1)^{F}\right)\right] \\
\int d l\left\langle D p, \epsilon\left|e^{-l H_{\text {closed }}}\right| D p,-\epsilon\right\rangle & =-\int \frac{d t}{t} \operatorname{Tr}_{R}\left[e^{-t H_{\text {open }}} \frac{1}{2}\left(1+(-1)^{F}\right)\right] .
\end{aligned}
$$

In particular, open strings between like D-branes are purely bosonic, whereas those between unlike D-branes (of equal $p$ ) are purely fermionic. The corresponding massless excitations are the appropriate dimensional reduction of a ten-dimensional vector and a ten-dimensional MajoranaWeyl spinor, respectively. For brane-antibrane amplitudes the sign in front of $(-1)^{F}$ is reversed. Thus the open string between a brane and an anti-brane of the same type contains a tachyon (but no massless vectors), and the open string between a brane of one type and an anti-brane of the other has fermions of the opposite chirality.

\subsection{Open strings}

The various Dirichlet-branes, and in particular the D9-branes, play a crucial role in the construction of the (open string) orientifold models. Although these models are somewhat peripheral to the main line of the paper, we shall nevertheless use the occasion to explain how they can be understood in terms of D-branes. The models fall into four classes, three of which are obtained from Type 0B, and one from Type 0A.

0B model 1: Like Type IIB string theory, Type 0B is invariant under the world-sheet parity operator $\Omega$, and we can therefore consider the corresponding projection. The massless string 
states that are invariant under $\Omega$ are the graviton, dilaton and the $\mathrm{R}$ - $\mathrm{R}$ 2-form in the untwisted sector, as well as the tachyon and the R-R 2-form in the twisted sector. The one-loop vacuum amplitude of the new theory has a contribution from the Klein bottle,

$$
K=\int \frac{d t}{2 t} \operatorname{Tr}_{\text {closed }}\left[e^{-t H_{\text {closed }}} \frac{1}{2}\left(1+(-1)^{F_{L}+F_{R}}\right) \frac{1}{2} \Omega\right]
$$

When interpreted in the tree channel, this gives rise to a massless NS-NS tadpole. The theory does not have a massless $\mathrm{R}-\mathrm{R}$ tadpole, as that would arise from an insertion of $\left((-1)^{F_{L}}+(-1)^{F_{R}}\right)$ in the trace. The presence of the massless NS-NS tadpole does not render the theory necessarily inconsistent; it only means that the vacuum has to be shifted by the Fischler-Susskind mechanism 18. Alternatively, one can introduce 64 D9-branes to cancel the NS-NS tadpole. However, as the net R-R charge must still vanish, 32 of them must be anti-D9-branes. Since there are two kinds of D9-branes in Type 0B, one can introduce $n$ of one kind and $32-n$ of the other, together with their respective anti-branes. From the analysis of the previous subsection we can easily determine the spectrum of open string in this theory. In particular, the gauge group is $(S O(n) \times S O(32-n))^{2}$; all the other low lying states are given in table 1.

For the specific choice $n=0$ ( or $n=32$ ), the resulting theory is purely bosonic, and has a gauge group $S O(32) \times S O(32)$. It is further singled out by the property that one of its D-strings possesses low-lying modes that reproduce the world-sheet theory of the $D=26$ bosonic string compactified on an $S O(32)$ lattice to ten dimensions. This has led to the suggestion that the two models might be related by a strong/weak coupling duality [0].

$\underline{\text { 0B model 2: }}$ The action of $\Omega$ can also be combined with another $\mathbb{Z}_{2}$ symmetry. In particular, we can consider the operator $\widehat{\Omega}$ that differs from $\Omega$ by a sign in the R-R sector [6], and can therefore be described as $\widehat{\Omega}=\Omega(-1)^{F_{R}^{s}}$, where $F_{R}^{s}$ is the right-moving part of the space-time fermion number operator. ( $\widehat{\Omega}$ is of order two since $\widehat{\Omega}^{2}=(-1)^{F^{s}} \equiv 1$ in Type 0B.) The invariant massless fields now include the two scalars and the 4 -form, instead of the two 2 -forms. The Klein bottle contribution to the vacuum amplitude is again given by $(2.16)$, with $\Omega$ replaced by $\widehat{\Omega}$, and therefore the massless R-R tadpole vanishes. The theory has a NS-NS tadpole, but it is tachyonic rather that massless. Since the theory has a closed string tachyon anyway, this is believed to be harmless, and there is therefore no need to introduce any branes at all. The resulting theory is thus a closed bosonic string theory whose low-lying spectrum is shown in table 1.

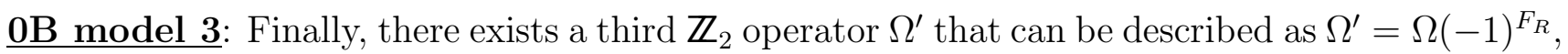
[6, 8, 9]. ( $\left(\Omega^{\prime}\right.$ is of order two since $\left(\Omega^{\prime}\right)^{2}=(-1)^{F_{L}+F_{R}} \equiv 1$ in Type 0B.) Because of the $(-1)^{F_{R}}$, this projection removes the tachyon. At the massless level, the surviving fields are the graviton and dilaton from the NS-NS sector, and a single scalar, a single 2-form, and a 4-form with antiself-dual field strength from the R-R sector. Replacing $\Omega$ by $\Omega^{\prime}$ in the Klein bottle (2.16) results in a trace with $\left((-1)^{F_{L}}+(-1)^{F_{R}}\right)$, and therefore the theory has a massless $\mathrm{R}-\mathrm{R}$ tadpole, but no massless NS-NS tadpole. For consistency, this tadpole must be cancelled by the introduction of D9-branes. Since the operator $(-1)^{F_{R}}$ maps one kind of D9-brane $(|D 9,+\rangle$ say) to the other 
$(|D 9,-\rangle)$ (see [7, 9]), we are forced to introduce them in pairs. In order to cancel the R-R tadpole, a net number of 32 D9-brane pairs is required. The inclusion of these D9-branes necessarily introduces a massless NS-NS tadpole which cannot be cancelled (but which, as before, does not render the theory necessarily inconsistent). The resulting gauge group is $U(32)$, and the theory is completely free of tachyons. More generally, since the total number of D9-branes is not restricted, one can add an arbitrary but equal number of D9-brane pairs and anti-D9-brane pairs to the background, without introducing a R-R tadpole. The resulting gauge group is then $U(n) \times U(32+n)$, where $n$ denotes the number of anti-D9-brane pairs. This theory has tachyons in the bi-fundamental and its complex conjugate.

0A model: Unlike its supersymmetric cousin, Type $0 \mathrm{~A}$ is also invariant under $\Omega$, and we can therefore consider its orientifold [6]. In the NS-NS sector, $\Omega$ acts in the same way as for Type $0 \mathrm{~B}$, and therefore leaves the graviton, dilaton, and tachyon invariant. In the R-R sector, $\Omega$ exchanges the two 1-forms and the two 3 -forms, and therefore only the symmetric combinations are invariant. The resulting theory has a Klein bottle given by (2.16), and therefore a massless NS-NS tadpole, but no R-R tadpole. This can be cancelled by the inclusion of open string states, leading to the gauge group $S O(n) \times S O(32-n)$, where $n \in\{1, \ldots, 32\}$. The open string spectrum also contains tachyons in the singlet representation, as well as in the symmetric tensor representations of either $S O$ factor. There are also massless fermions in the bi-vector representation. In terms of D-branes, these open strings can be understood to originate from $n$ unstable D9-branes of one type $(|\widehat{D 9},+\rangle$, say) and $32-n$ unstable D9-branes of the other type $(|\widehat{D 9},-\rangle)$. The tachyons arise from open strings which begin and end on like D9-branes (and in particular open strings beginning and ending on the same D9-brane), and the massless fermions arise from open strings which stretch between unlike D9-branes. These 9-branes only carry NS-NS charge and can therefore cancel the NS-NS tadpole.

Unlike in the case of Type 0B, the operators $\widehat{\Omega}=\Omega(-1)^{F_{R}^{S}}$ and $\Omega^{\prime}=\Omega(-1)^{F_{R}}$ do not give new open string models when applied to Type 0A. The former gives precisely the same Type 0A model as before, except that now only the anti-symmetric combinations of the 1-forms and 3-forms survive. The latter operator generates a $\mathbb{Z}_{4}$ rather than $\mathbb{Z}_{2}$ symmetry, since $\Omega^{\prime 2}=(-1)^{F_{L}+F_{R}}$ is +1 in the NS-NS sector, but -1 in the R-R sector. The orbifold by $(-1)^{F_{L}+F_{R}}$ gives Type 0B, and the resulting model is therefore one of the Type 0B open string theories.

\section{$3 \quad$ From M-theory to Type 0}

Given the relation of Type 0A string theory to Type IIA, one may ask whether the strong coupling dynamics of the former is also eleven-dimensional in character. The obvious candidate for such an eleven-dimensional theory is the orbifold of M-theory by $(-1)^{F^{s}}$. Type 0A would then correspond to the compactification of this orbifold on a circle of radius $R^{(M)}$; a simple argument, however, rules this scenario out. 


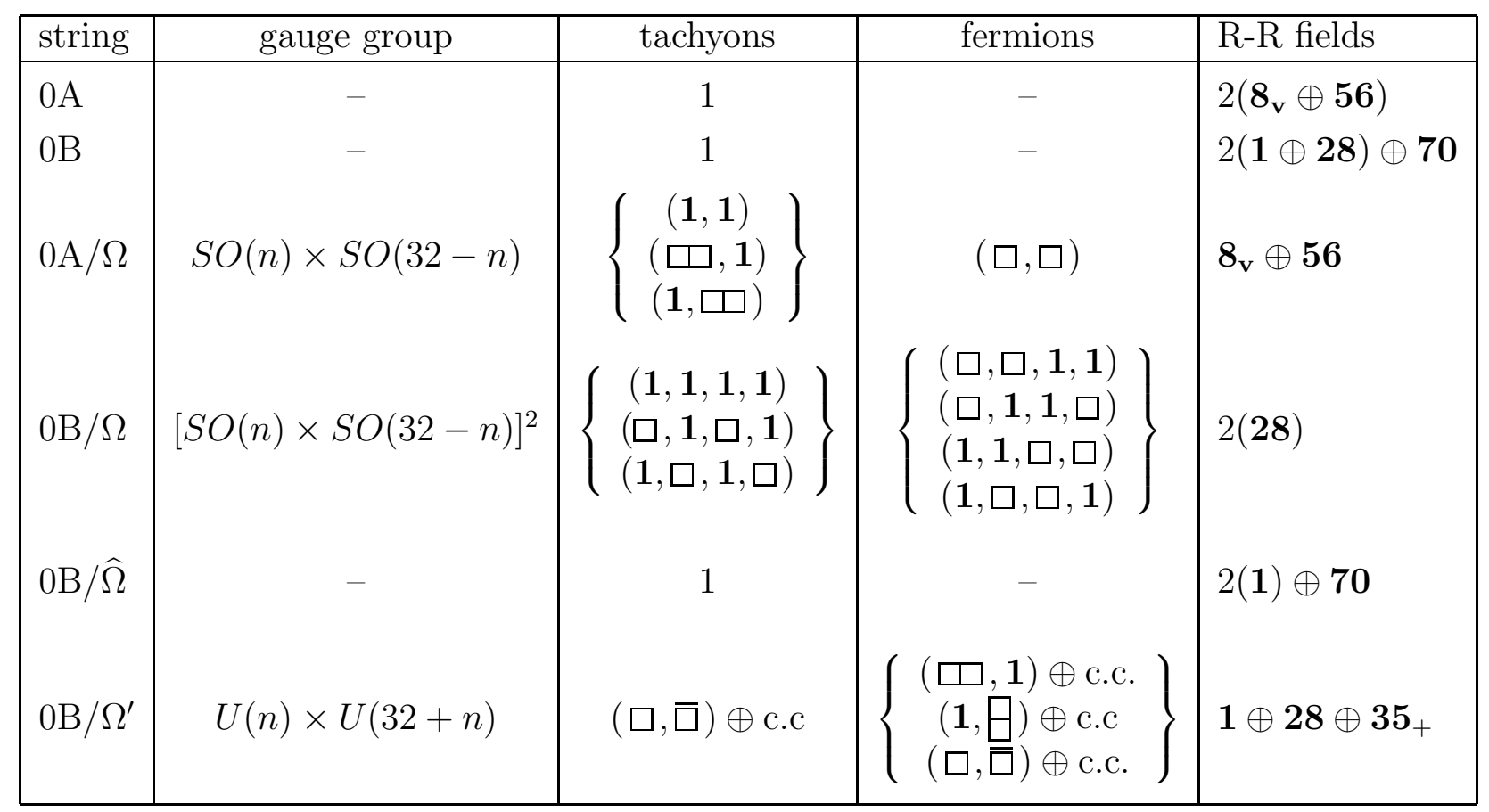

Table 1: Type 0 string theories - low-lying spectrum.

In the limit $R^{(M)} \rightarrow 0$, the M-theory orbifold should reproduce the massless spectrum of Type $0 \mathrm{~A}$, and therefore in particular two 1-forms, a 2-form, and two 3-forms. Since the action of the orbifold is independent of $R^{(M)}$, these can only arise by Kaluza-Klein reduction of massless fields in eleven dimensions. The invariant sector of the original massless eleven-dimensional supergravity multiplet consists of the graviton and the 3-form, which upon Kaluza-Klein reduction are only able to produce a single 1-form, a 2-form, and a single 3-form. The additional 1-form and 3-form must come from a 'twisted sector' of the orbifold. For the 1-form this requires either a massless 1-form or 2-form in eleven dimensions, and for the 3-form it requires either a massless 3-form or 4-form. In either case, this would lead to additional massless states in ten dimensions that are not present in Type 0A. Therefore Type 0A cannot correspond to the circle compactification of $\mathrm{M} /(-1)^{F^{s}}$; the same argument actually implies more generally that Type 0A cannot be obtained by ordinary Kaluza-Klein dimensional reduction from any theory in eleven dimensions.

\subsection{Smooth orbifolds}

It is however possible to obtain insight into the relation between Type 0A and M-theory by smoothing out the orbifold which produces Type 0A from Type IIA. The general prescription 
for doing this is to compactify an extra dimension on a circle of radius $R$, and combine the action of the original discrete symmetry with that of a half-shift along the circle. If we denote the original $\left(\mathbb{Z}_{2}\right)$ symmetry by $g$, the new symmetry is given by $g \cdot S$, where $S$ denotes a halfshift along the circle. The untwisted sector corresponds to strings with integer winding number, and consists of $g$-even states with even Kaluza-Klein momentum, and $g$-odd states with odd Kaluza-Klein momentum. In addition, there is a twisted sector arising from $g$-twisted strings of half-odd-integer winding number, in which the states are again $g$-even with even momentum, and $g$-odd with odd momentum. In the limit $R \rightarrow 0$, the $g$-odd states of both the untwisted and the twisted sector decouple, and states with different winding numbers become degenerate. In this limit we therefore reproduce the spectrum of the original orbifold by $g$ compactified on a circle of vanishing radius (or, equivalently the T-dual of this orbifold at infinite radius).

For $g=(-1)^{F^{s}}$ the resulting nine-dimensional theories obtained from Type IIA and Type IIB were first considered in [19], and are related by a Wick rotation to the finite temperature strings considered in [20]. Their spectrum is given by

$$
\begin{array}{clll} 
& (N S+, N S+) \oplus(R+, R \mp), & p_{9}=2 m / R_{9}^{(I I)}, & w_{9}=n R_{9}^{(I I)} \\
\text { untwisted: } & (R+, N S+) \oplus(N S+, R \mp), & p_{9}=(2 m+1) / R_{9}^{(I I)}, & w_{9}=n R_{9}^{(I I)} \\
& (N S-, N S-) \oplus(R-, R \pm), & p_{9}=2 m / R_{9}^{(I I)}, & \\
\text { twisted: } & (R-, N S-) \oplus(N S-, R \pm), & p_{9}=(2 m+1) / R_{9}^{(I I)}, & w_{9}=(n+1 / 2) R_{9}^{(I I)} \\
& (R-1 / 2) R_{9}^{(I I)},
\end{array}
$$

where the upper (lower) sign corresponds to Type IIA(IIB). In the limit $R_{9}^{(I I)} \rightarrow \infty$ one regains the spectrum of ten-dimensional Type IIA(B) (2.1), and in the limit $R_{9}^{(I I)} \rightarrow 0$ one obtains the spectrum of Type $0 \mathrm{~A}(\mathrm{~B})$ for $R_{9}^{(0)}=0$ (2.5). In particular, all the fermions are removed, and additional R-R fields become massless in this limit. In addition, there is a tachyon in the twisted sector when $R_{9}^{(I I)}<\sqrt{2}$. For $R_{9}^{(I I)}>\sqrt{2}$ these theories have a finite vacuum energy, which decreases monotonically with $R_{9}^{(I I)}$, and vanishes in the supersymmetric limit $R_{9}^{(I I)} \rightarrow \infty$ [19. In this regime, the nine-dimensional theories therefore flow back to the ten-dimensional Type II theories. When $R_{9}^{(I I)}<\sqrt{2}$, on the other hand, the vacuum energy diverges due to the presence of the tachyon. The theories are therefore either inconsistent in this regime, or they undergo a phase transition (akin to the Hagedorn transition at finite temperature), by tachyon condensation, into a stable vacuum.

\subsection{Type 0A}

Strictly speaking, the above orbifold procedure is reliable only at weak coupling, for which the corresponding spectrum can be trusted; we can therefore identify

$$
\text { Type IIA on } \mathbf{S}^{1} /\left.(-1)^{F^{s}} \cdot S\right|_{g^{(I I A)}=0, R_{9}^{(I I A)}=0}=\text { Type } \mathbf{0 A} \text { on }\left.\mathbf{S}^{1}\right|_{g^{(0 A)}=0, R_{9}^{(0 A)}=0},
$$


and similarly for Type IIB. On the other hand Type IIA string theory corresponds to the compactification of M-theory on a circle. From the point of view of M-theory, the above ninedimensional theory thus corresponds to the compactification on $\mathbf{S}^{1} \times \mathbf{S}^{1} /(-1)^{F^{s}} \cdot S$, where both circles are small. Let us denote the radius of the ordinary circle (when measured with respect to the eleven-dimensional metric) by $R_{10}^{(M)}$, and the radius of the orbifolded circle by $R_{9}^{(M)}$. The (ten-dimensional) coupling constant and radius in the nine-dimensional theory are then given by

$$
g^{(I I A)}=\left(M_{11} R_{10}^{(M)}\right)^{3 / 2}, \quad R_{9}^{(I I A)}=\left(M_{11} R_{10}^{(M)}\right)^{1 / 2} R_{9}^{(M)},
$$

where $M_{11}$ is the eleven-dimensional Planck mass. By exchanging the roles of the two circles (see Fig. 1(a)), we can describe this theory equivalently as an ordinary circle compactification of a ten-dimensional theory. The identification (3.2) for zero coupling and radius suggests then that this ten-dimensional theory is Type 0A, where the coupling constant and radius are given by

$$
g^{(0 A)}=\left(M_{11} R_{9}^{(M)} / 2\right)^{3 / 2}, \quad R_{9}^{(0 A)}=\left(M_{11} R_{9}^{(M)} / 2\right)^{1 / 2} R_{10}^{(M)} .
$$

We are thus led to conjecture that

$$
\text { Type 0A }=\text { M-theory on } \mathbf{S}^{1} /(-1)^{F^{s}} \cdot S .
$$

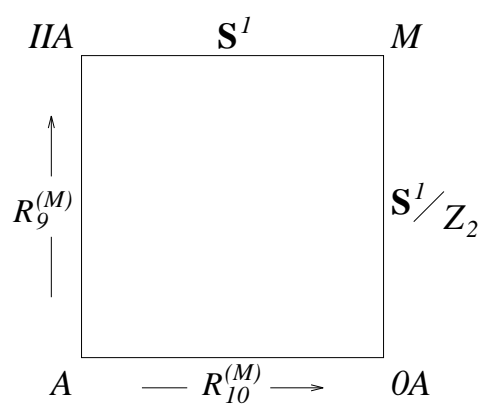

a

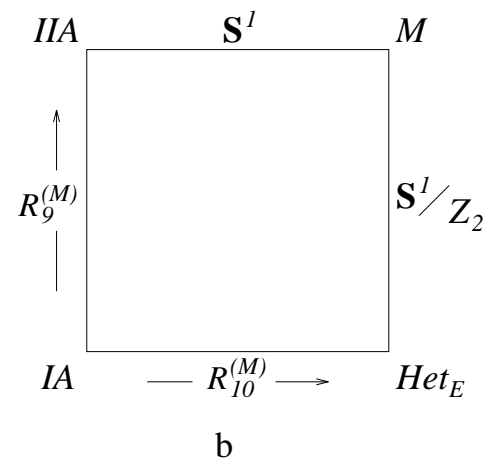

$\mathrm{b}$

Figure 1: The '9-10 flip' in M-theory; (a) Type 0A, (b) Heterotic $E_{8} \times E_{8}$.

In essence, the conjecture means that Type 0A string theory corresponds to M-theory compactified on a circle of radius $R^{(M)} / 2$, where we impose periodic boundary conditions for bosons, and anti-periodic boundary conditions for fermions.] The massless spectrum of the orbifold contains the bosonic part of the Type IIA supergravity multiplet, namely a ten-dimensional graviton, a Kalb-Ramond field and a dilaton, together with a (single) 1-form field and a 3-form field. The

\footnotetext{
${ }^{6}$ The factor of $1 / 2$ is due to the half-shift $S$.

${ }^{7}$ A Euclidean version of Type $0 \mathrm{~A}$ can then be thought of as M-theory at finite temperature, where $T \sim 1 / R^{(M)}$.
} 
action of the orbifold symmetry does not have any fixed points, and the resulting theory therefore corresponds to a closed string theory (if it defines a string theory at all). Since the orbifold also breaks supersymmetry, this closed string theory can only be either Type 0A or Type 0B, or one of the non-supersymmetric heterotic theories. As we have just seen, the massless spectrum contains the bosonic part of the Type IIA supergravity multiplet, and therefore the only possibility is Type 0A.

M-theory orbifolds, like string theory orbifolds, usually require the inclusion of 'twisted sector' states for consistency [21, 22, 23]. However, unlike string theory where twisted sectors arise from strings that close up to the action by an element of the orbifold group, the origin of twisted sectors in M-theory orbifolds is much less understood. Instead, the twisted sectors of M-theory orbifolds are usually determined by indirect arguments such as the requirement that certain symmetries are protected from anomalies. (This is actually somewhat analogous to the way in which twisted sectors of asymmetric string orbifolds are determined by the requirement of modular invariance.) The paradigm for this is the compactification of M-theory on $\mathbf{S}^{1} / \mathbb{Z}_{2}$ [21], where the non-trivial element of the orbifold group reflects the compact coordinate and reverses the sign of the 3 -form,

$$
x^{10} \rightarrow-x^{10}, \quad C^{(3)} \rightarrow-C^{(3)} .
$$

If we compactify the theory on an additional circle to nine dimensions and exchange the roles of the two compact directions, similar arguments as above suggest that the M-theory orbifold corresponds to the $E_{8} \times E_{8}$ heterotic string (see Fig. 1(b)). Indeed, the untwisted sector of the M-theory orbifold accounts for the $N=1$ gravity supermultiplet, and the twisted sectors provide the two $E_{8}$ gauge multiplets. These can also be shown to be necessary in order to cancel the gravitational anomaly due to the boundaries. In the present case, however, the orbifold by $(-1)^{F^{s}} \cdot S$ does not introduce a gravitational anomaly, and it is therefore not clear what symmetry would be violated if the twisted sector were absent.

In the limit $R^{(M)} \rightarrow 0$ the twisted sector of our orbifold should include a tachyon and an additional massless 1 -form and 3 -form field. This is now possible, in principle, since the action of the orbifold does depend on $R^{(M)}$, and so fields which are massive at large $R^{(M)}$ may become massless, or even tachyonic, when $R^{(M)} \rightarrow 0$. Conversely, the additional $\mathrm{R}-\mathrm{R}$ fields of Type $0 \mathrm{~A}$ should become massive at non-zero coupling, and the tachyon should become massive at sufficiently strong coupling. Therefore the tachyonic instability disappears in the strong coupling regime, and the theory proceeds to flow back to eleven dimensions.

\section{$3.3 \quad$ T-duality and Type 0B}

It is well known that the $\mathbf{S}^{1}$ compactifications of Type IIA and Type IIB string theories are related by T-duality; this symmetry inverts the radius of the circle, exchanges winding and momentum states, and changes the sign of $(-1)^{F_{R}}$ in the right-moving R-sector. Similarly, T-duality on a circle relates Type $0 \mathrm{~A}$ and Type $0 \mathrm{~B}$ to one another. 
The transformation of the nine-dimensional theories described in subsection 3.1 under Tduality is somewhat more complicated. T-duality still exchanges winding and momentum states, and this requires that it maps $R_{9}^{(I I)} \rightarrow 2 / R_{9}^{(I I)}$ so that even momentum becomes integer winding, and odd momentum becomes half-odd-integer winding. In the T-dual theory all spacetime bosons therefore have integer winding, whereas all spacetime fermions have half-odd-integer winding. Furthermore, states which are even under $(-1)^{F_{R}}$ have even momentum, and those odd under $(-1)^{F_{R}}$ carry odd momentum. It thus follows that the theory T-dual to Type IIA (IIB) on $\mathbf{S}^{1} /(-1)^{F^{s}} \cdot S$ is given by Type 0B $(0 \mathrm{~A})$ on $\mathbf{S}^{1} /(-1)^{F_{R}} \cdot S$ (compare also [24]).

The '9-10 flip' (Fig. 1(a)), which allowed us to identify Type 0A as M-theory on $\mathbf{S}^{1} /(-1)^{F^{s}} \cdot S$, is equivalent to the sequence of duality transformations given by $T^{\prime} \circ S \circ T$ [25], where

$$
\begin{array}{rll}
T: & R \rightarrow 1 / R, & g \rightarrow g / R \\
T^{\prime}: & R \rightarrow 2 / R, & g \rightarrow g / R \\
S: & R \rightarrow g^{-1 / 2} R, & g \rightarrow 1 / g .
\end{array}
$$

It therefore implies an S-duality relation between Type 0B on $\mathbf{S}^{1}$ and Type 0B on $\mathbf{S}^{1} /(-1)^{F_{R}} \cdot S$. In the infinite radius limit this reduces to the statement that Type $0 \mathrm{~B}$ is symmetric under $\mathrm{S}$ duality.

The T-duality relation between Type $0 \mathrm{~A}$ and Type $0 \mathrm{~B}$ suggests that the tachyon of Type 0B becomes massive when $g^{(0 B)} / R^{(0 B)}$ is sufficiently large. In this regime, the theory behaves differently depending on whether $g^{(0 B)} \gg 1$ and $R^{(0 B)}$ is fixed, or whether $R^{(0 B)} \ll 1$ and $g^{(0 B)}$ is fixed. In the first limit, the theory is related by S-duality to Type 0B on $\mathbf{S}^{1} /(-1)^{F_{R}} \cdot S$, with a weak coupling and small radius given by

$$
g^{\prime(0 B)}=1 / g^{(0 B)} \ll 1, \quad R^{\prime(0 B)}=R^{(0 B)} / \sqrt{g^{(0 B)}} \ll 1 .
$$

This is in turn related by T-duality to Type IIA on $\mathbf{S}^{1} /(-1)^{F^{s}} \cdot S$ at weak coupling and large radius given by

$$
g^{(I I A)}=1 /\left(\sqrt{g^{(0 B)}} R^{(0 B)}\right) \ll 1, \quad R^{(I I A)}=2 \sqrt{g^{(0 B}} / R^{(0 B)} \gg 1 .
$$

Type 0B therefore flows to weakly coupled ten-dimensional Type IIA in this limit. In the second limit, the theory is related by T-duality to Type 0A at large radius and strong coupling,

$$
g^{(0 A)}=g^{(0 B)} / R^{(0 B)} \gg 1, \quad R^{(0 A)}=1 / R^{(0 B)} \gg 1
$$

which, as we have already seen, flows to eleven-dimensional M-theory.

In terms of M-theory, again because of T-duality, Type 0B corresponds to

$$
\text { Type } \mathbf{0 B}=\text { M-theory on } \mathbf{T}^{2} /\left.(-1)^{F^{s}} \cdot S\right|_{V=0},
$$

\footnotetext{
${ }^{8} \mathrm{We}$ are assuming here that T-duality is a symmetry beyond the perturbative regime.
} 
where the shift acts on one of the cycles of the torus, and the coupling constant is identified with the imaginary part of the complex structure of the torus. This then suggests that Type 0B is symmetric not only under S-duality, but under the full $S L(2, \mathbb{Z})$ symmetry.

In Type IIB S-duality exchanges the fundamental string with the D-string, and likewise the NS-NS 2-form with the R-R 2-form. The spectrum of open strings that begin and end on the D-string contains 8 massless bosons and 8 massless fermions of either chirality, and these agree precisely with the low-lying excitations of the fundamental string.

In Type 0B there are two D-strings, $|D 1,+\rangle$ and $|D 1,-\rangle$, and correspondingly two R-R 2-forms. Neither of these D-strings has the correct degrees of freedom to correspond to a fundamental Type 0B string, as neither possesses fermionic zero modes, which would correspond to the world-sheet fermions of the fundamental string. Instead, the object that is S-dual to the fundamental string corresponds to a combination of both types of D-strings. [ The fermions are then provided by open strings which begin on one D-string and end on the other. It follows from (2.14) that the combination is only charged under the untwisted R-R fields, and therefore that S-duality exchanges the NS-NS 2-form with the R-R 2-form in the untwisted sector. The twisted fields are invariant under the duality.

Such a combination should appear as a bound state of the two D-strings. In the following section we shall argue that an analogous bound state of the two different Type 0A D-particles forms in the regime where the tachyon becomes massive. This suggests that the same is true for the two different Type 0B D-strings, namely that they form a bound state in nine-dimensions when $g^{(0 B)} / R^{(0 B)}$ is large enough to make the tachyon massive. The ten-dimensional picture is somewhat less clear.

\section{Type 0A fermions}

In the M-theory orbifold the fermions carry odd Kaluza-Klein momentum, and therefore have a minimal (classical) mass

$$
m_{f}=\frac{1}{R^{(M)}} .
$$

In the limit $R^{(M)} \rightarrow 0$ the fermions decouple, and we obtain a purely bosonic spectrum. However, as in the case of ordinary Kaluza-Klein excitations of M-theory on $\mathbf{S}^{1}$, the existence of states with mass (4.1) suggests that Type 0A has corresponding non-perturbative (Dirichlet-brane-like) states whose mass is proportional to $1 / g^{(0 A)}$. In particular, eq. (4.1) implies that Type 0A (which perturbatively is a purely bosonic theory) contains fermionic solitons.

Strictly speaking, the above expression for the mass can only be trusted in the classical limit, i.e. for $R^{(M)} \gg M_{11}^{-1}$. Due to the lack of supersymmetry, masses are not protected against

\footnotetext{
${ }^{9}$ Such a combination has been discussed in the context of D3-branes in [2], where it was termed a 'self-dual' 3-brane.
} 
quantum corrections at small $R^{(M)}$. In fact, since there is a tachyon in the spectrum, one expects that the masses are renormalized by an infinite amount. As a consequence, one should not expect to find any fermions in weakly coupled Type 0A string theory; the fermions should only appear when the coupling constant is sufficiently large to remove the tachyon.

However as we shall now explain, all the ingredients that are necessary to form these fermions are already present in weakly coupled Type 0A theory. Recall that the theory has two kinds of D-particles, $|D 0,+\rangle$ and $|D 0,-\rangle$. Since open strings beginning and ending on the same D-particle are purely bosonic, each of the D-particles is bosonic, and has a non-degenerate ground state. Strings beginning on $|D 0,+\rangle$ and ending on $|D 0,-\rangle$, on the other hand, are purely fermionic. A configuration of two coincident D-particles of different type would therefore possess fermionic zero-modes, whose quantization would result in a supermultiplet ground state, and thus spacetime fermions. More realistically, the two D-particles might form a bound state with a non-vanishing separation. The fermionic modes would then be massive, and this would lead to a non-degenerate bosonic ground state and at least one stable fermionic excited state.

We shall now argue that such a bound state can form when the tachyon becomes massive. To lowest order, the potential between unlike D-particles is given in the open string (loop) channel by

$$
V_{+-}(r)=2 \int \frac{d t}{2 t} \int \frac{d k^{0}}{2 \pi} \operatorname{Tr}_{R}\left[e^{-2 \pi \alpha^{\prime} t\left(k^{2}+M^{2}(r)\right)} \frac{1}{2}\left(1+(-1)^{F}\right)\right],
$$

where $r$ is the separation between the D-particles. Upon integration of $k^{0}$, we find

$$
V_{+-}(r)=\int \frac{d t}{2 t}\left(8 \pi^{2} \alpha^{\prime} t\right)^{-1 / 2} e^{-t r^{2} /\left(2 \pi \alpha^{\prime}\right)} \frac{f_{2}^{8}(q)}{f_{1}^{8}(q)},
$$

where $f_{i}$ are the standard $f$ functions [5], and $q=\exp (-2 \pi t)$. At short distances, $r \ll \sqrt{\alpha^{\prime}}$, we can approximate $V_{+-}$by taking the limit $t \rightarrow \infty$, in which case

$$
\frac{f_{2}^{8}(q)}{f_{1}^{8}(q)} \sim 16+O\left(e^{-\pi t}\right) .
$$

In this approximation, the potential becomes $\square$

$$
V_{+-}^{\text {short }}(r) \sim \frac{8}{\sqrt{8 \pi^{2} \alpha^{\prime}}} \int \frac{d t}{t^{3 / 2}} e^{-t r^{2} /\left(2 \pi \alpha^{\prime}\right)}=-\frac{4}{\pi \alpha^{\prime}}|r| .
$$

In contrast, the potential between like D-particles is given by the open string amplitude

$$
\begin{aligned}
V_{++}(r) & =-2 \int \frac{d t}{2 t} \int \frac{d k^{0}}{2 \pi} \operatorname{Tr}_{N S}\left[e^{-2 \pi \alpha^{\prime} t\left(k^{2}+M^{2}(r)\right)} \frac{1}{2}\left(1+(-1)^{F}\right)\right] \\
& =-\int \frac{d t}{2 t}\left(8 \pi^{2} \alpha^{\prime} t\right)^{-1 / 2} e^{-t r^{2} /\left(2 \pi \alpha^{\prime}\right)} \frac{f_{3}^{8}(q)-f_{4}^{8}(q)}{f_{1}^{8}(q)}
\end{aligned}
$$

\footnotetext{
${ }^{10}$ The expression for the potential is similar to the D0-D8 case in Type IIA [26], and is evaluated using the analytic continuation of the gamma function to negative argument.
} 
which at short distances becomes

$$
V_{++}^{\text {short }}(r) \sim \frac{4}{\pi \alpha^{\prime}}|r| .
$$

We therefore find that the potential between like D-particles is attractive at short distances, whereas the potential between unlike D-particles is repulsive.

At long distances the picture is complicated by the tachyon. For $r \gg \sqrt{\alpha^{\prime}}$ we can approximate the amplitude by taking the limit $t \rightarrow 0$, where

$$
\frac{f_{2}^{8}(q)}{f_{1}^{8}(q)}=\frac{f_{3}^{8}(q)-f_{4}^{8}(q)}{f_{1}^{8}(q)} \sim t^{4}\left(e^{\pi / t}-8+O\left(e^{-\pi / t}\right)\right) .
$$

The first dominant term corresponds to the exchange of the closed string tachyon, and gives a divergent contribution to the potential. It is attractive for like D-particles and repulsive for unlike ones. The second term corresponds to the exchange of massless fields, and gives a repulsive contribution for like D-particles and an attractive one for unlike D-particles. Indeed, like Dparticles interact through massless R-R fields as well as through massless NS-NS fields (gravity and dilaton). The former is repulsive, and twice as strong as the latter, which is attractive. The net interaction due to massless fields is therefore repulsive. Unlike D-particles, on the other hand, are charged under different R-R fields, and so interact only through the massless NS-NS fields; their interaction is therefore attractive at the massless level.

As we have learned in the previous section, at strong coupling the Type 0A tachyon acquires a mass, whereas the massless NS-NS fields remain massless. Although we cannot directly trust either the short or the long distance calculation of the potential at strong coupling, the long distance force will be dominated by the exchange of the lowest-lying closed string states, and this implies, whatever the details, that the long-distance potential between two different D0-branes is attractive at strong Type 0A coupling. Thus at strong 0A coupling, the bound state is likely to exist, and it has the right properties to correspond to the fermionic KK excitations of the M-theory orbifold.

\section{Non-perturbative consistency of open string models}

The Type 0 open string models of table 1 were constructed by projecting Type 0A and Type 0B string theory either by the world-sheet parity operator $\Omega$, or by a combination of $\Omega$ with another $\mathbb{Z}_{2}$ symmetry. Given our conjecture about the eleven-dimensional origin of Type $0 \mathrm{~A}$ and Type $0 \mathrm{~B}$, it is worthwhile to re-examine these models in the context of M-theory.

It is well known that $\Omega$ lifts to the operation that inverts the sign of the 3 -form. This by itself is not a symmetry of M-theory as it reverses the orientation of the membrane. The Type 0A open string model can therefore not be related to M-theory, and is thus inconsistent at non-zero coupling. Another way of reaching the same conclusion is to observe that (at zero coupling) $\Omega$ 
exchanges the massless R-R fields in the untwisted sector of Type 0A with those in the twisted sector. As the coupling is increased the twisted fields become massive, and $\Omega$ does not define a symmetry any more. This again implies that the corresponding open string model is only consistent at zero coupling.

The consistency of the Type 0B models is most easily analysed by considering their T-dual Type 0A models after compactification on a circle. There are three cases to consider. The orientifold of Type $0 \mathrm{~B}$ by $\Omega$ is related by T-duality to Type $0 \mathrm{~A}$ on $\mathbf{S}^{\mathbf{1}} / \Omega \cdot I$, where $I$ denotes a reflection in the compact direction. In terms of M-theory this projection corresponds to the symmetry (3.5), and therefore the model is presumably consistent for all values of the coupling constant.

The T-dual of the second orientifold model of Type 0B by $\widehat{\Omega}=\Omega(-1)^{F_{R}^{s}}$ is Type 0A on $\mathbf{S}^{1} / \Omega(-1)^{F_{R}^{s}} \cdot I$. This differs from the first orientifold projection by the operator $(-1)^{F_{R}^{s}}$, that acts as $+1(-1)$ on all states in the untwisted NS-NS (R-R) sector of Type 0A. This does not lift to a symmetry of M-theory, and therefore the associated orientifold model is inconsistent at finite coupling.

Finally, the third Type 0B model, involving $\Omega^{\prime}=\Omega(-1)^{F_{R}}$, is related by T-duality to Type $0 \mathrm{~A}$ on $\mathbf{S}^{\mathbf{1}} / \Omega(-1)^{F_{R}} \cdot I$. This differs from the first orientifold projection by $(-1)^{F_{R}}$, that acts as $+1(-1)$ on the untwisted (twisted) sector of the M-theory orbifold. This presumably defines a symmetry of M-theory, but since we do not have detailed knowledge of the twisted sector, we cannot check this further.

\section{Conclusions}

In this paper we have argued that two of the non-supersymmetric closed string theories in ten dimensions, Type 0A and Type 0B, fit into the framework of M-theory. We have shown that the spectrum of Type 0A agrees, in a certain limit, with that of the orbifold of M-theory on $\mathbf{S}^{1} /(-1)^{F^{s}} \cdot S$. We have also explained how the fermionic states of the M-theory orbifold might be accounted for in terms of D-particles in Type 0A. One consequence of this conjecture is that the tachyon of Type 0A becomes massive at strong coupling. The conjecture also implies that Type 0B is self-dual, and that some of the previously obtained Type 0 open string models are inconsistent at finite coupling.

A crucial role in our discussion was played by the nine-dimensional theory that interpolates between Type IIA at infinite radius and Type 0A at zero radius. This theory has a non-vanishing one-loop cosmological constant which diverges at the radius where the tachyon appears [19]. The size of the circle is therefore not a modulus of the theory, and there exists a non-trivial potential which is minimized at infinite radius; for sufficiently large radii the theory therefore decompactifies. This suggests that the same is true for the ten-dimensional interpolating theory, and therefore that Type 0A will decompactify dynamically to eleven dimension at sufficiently 
strong coupling.

It would be interesting to understand how the other non-supersymmetric theories fit into the M-theory framework. Some time ago we argued that one of the orientifolds of Type 0B is dual to the compactification of the 26-dimensional bosonic string on the $S O(32)$ weight lattice [7], but the relations of the other non-supersymmetric theories to one another (and to M-theory) remain to be understood. Some progress in this direction has also been made in [11], but much still remains to be uncovered.

\section{Acknowledgements}

We would like to thank Igor Klebanov for very useful conversations, and Ralph Blumenhagen and Shigeki Sugimoto for pointing out a couple of errors in an earlier version of this paper. O.B. is supported in part by the DOE under grant no. DE-FG03-92-ER 40701. M.R.G. is supported by a College Lectureship of Fitzwilliam College, Cambridge.

\section{References}

[1] L.J. Dixon, J.A. Harvey, String theories in ten dimensions without spacetime supersymmetry, Nucl. Phys. B274, 93 (1986).

N. Seiberg, E. Witten, Spin structures in string theory, Nucl. Phys. B276, 272 (1986).

H. Kawai, D.C. Lewellen, S.-H. H. Tye, Classification of closed-fermionic-string models, Phys. Rev. D 34, 3794 (1986).

[2] I.R. Klebanov, A.A. Tseytlin, D-branes and dual gauge theories in type 0 Strings, Nucl. Phys. B546, 155 (1999); hep-th/9811035.

I.R. Klebanov, A.A. Tseytlin, Asymptotic freedom and infrared behaviour in the type 0 string approach to gauge theory, Nucl. Phys. B547, 143 (1999); hep-th/9812089.

I.R. Klebanov, A.A. Tseytlin, A non-supersymmetric large $N$ CFT from Type 0 string theory, JHEP 9903, 015 (1999); hep-th/9901101.

[3] J.A. Minahan, Glueball mass spectra and other issues for supergravity duals of QCD models, JHEP 9901, 020 (1999); hep-th/9811156.

J.A. Minahan, Asymptotic freedom and confinement from Type 0 string theory, JHEP 9904, 007 (1999); hep-th/9902074.

I.I. Kogan, G. Luzon, Scale Invariance of Dirac Condition $g_{e} g_{m}=1$ in Type 0 String Approach to Gauge Theory, hep-th/9902086.

A.A. Tseytlin, K. Zarembo, Effective potential in non-supersymmetric $S U(N) \times S U(N)$ gauge theory and interactions of type 0 D3-branes, hep-th/9902095. 
N. Nekrasov, S.L. Shatashvili, On non-supersymmetric CFT in four dimensions, hepth/9902110.

M. Billo, B. Craps, F. Roose, On D-branes in Type 0 string theory, hep-th/9902196.

M. Alishahiha, A. Brandhuber, Y. Oz, Branes at singularities in Type 0 string theory, hep-th/9903186.

[4] J. Maldacena, The large $N$ limit of superconformal field theories and supergravity, Adv. Theor. Math. Phys. 2, 231 (1998); hep-th/9711200.

[5] J. Polchinski, Y. Cai, Consistency of open superstring theories, Nucl. Phys. B296, 91 (1988).

C.G. Callan, C. Lovelace, C.R. Nappi, S.A. Yost, Loop corrections to superstring equations of motion, Nucl. Phys. B308, 221 (1988).

[6] M. Bianchi, A. Sagnotti, On the systematics of open string theories, Phys. Lett. B247, 517 (1990).

M. Bianchi, A. Sagnotti, Twist symmetry and open-string Wilson lines, Nucl. Phys. B361, 519 (1991).

A. Sagnotti, Some properties of open-string theories, hep-th/9509080.

A. Sagnotti, Surprises in open string perturbation theory, hep-th/9702093.

[7] O. Bergman, M.R. Gaberdiel, A non-supersymmetric open string theory and S-duality, Nucl. Phys. B499, 183 (1997); hep-th/9701137.

[8] C. Angelantonj, Non-tachyonic open descendants of the type OB string theory, Phys. Lett. B444, 309 (1998); hep-th/9810214.

[9] R. Blumenhagen, A. Font, D. Lüst, Tachyon-free orientifolds of type OB strings in various dimensions, hep-th/9904069.

[10] A. Sen, An introduction to non-perturbative string theory, hep-th/9802051.

[11] J.D. Blum, K.R. Dienes, Duality without supersymmetry: the case of the $S O(16) \times S O(16)$ string, Phys. Lett. B414, 260 (1997); hep-th/9707148.

J.D. Blum, K.R. Dienes, Strong/weak coupling duality relations for non-supersymmetric string theories, Nucl. Phys. B516, 83 (1998); hep-th/9707160.

[12] A. Sen, Stable non-BPS states in string theory, JHEP 9806, 007 (1998); hep-th9803194. A. Sen, Stable non-BPS bound states of BPS D-branes, JHEP 9808, 010 (1998); hepth/9805019.

A. Sen, Tachyon condensation on the brane antibrane system, JHEP 9808, 012 (1998); hep-th/9805170. 
A. Sen, SO(32) Spinors of Type I and other solitons on brane-antibrane pair, JHEP 9809, 023 (1998); hep-th/9808141.

A. Sen, Type I D-particle and its interactions, JHEP 9810, 021 (1998); hep-th/9809111.

A. Sen, BPS D-branes on non-supersymmetric cycles, JHEP 9812, 021 (1998); hepth/9812031.

[13] O. Bergman, M.R. Gaberdiel, Stable non-BPS D-particles, Phys. Lett. B441, 133 (1998); hep-th/9806155.

O. Bergman, M.R. Gaberdiel, Non-BPS States in Heterotic - Type IIA Duality, JHEP 9903, 013 (1999); hep-th/9901014.

[14] E. Witten, D-branes and K-theory, JHEP 9812, 019 (1998); hep-th/9810188.

[15] M. Frau, L. Gallot, A. Lerda, P. Strigazzi, Stable non-BPS D-branes in type I string theory, hep-th/9903123.

[16] A. Sen, Non-BPS States and Branes in String Theory, hep-th/9904207.

[17] P. Horava, Type IIA D-branes, K-theory, and matrix theory, Adv. Theor. Math. Phys. 2, 1373 (1998); hep-th/9812135.

[18] W. Fischler, L. Susskind, Dilaton tadpoles, string condensates and scale invariance, I and II, Phys. Lett. B171, 383 (1986) and Phys. Lett. B173, 262 (1986).

[19] R. Rohm, Spontaneous supersymmetry breaking in supersymmetric string theories, Nucl. Phys. B237, 553 (1984).

[20] J.J. Atick, E. Witten, The Hagedorn transition and the number of degrees of freedom of string theory, Nucl. Phys. B310, 291 (1988).

[21] P. Horava, E. Witten Heterotic and Type I string dynamics from eleven dimensions, Nucl. Phys. B460, 506 (1996); hep-th/9510209.

[22] K. Dasgupta, S. Mukhi, Orbifolds of M-theory, Nucl. Phys. B465, 399 (1996); hepth/9512196.

[23] E. Witten, Five-branes and M-theory on an orbifold, Nucl. Phys. B463, 383 (1996); hepth/9512219.

[24] M.B. Green, Wilson-Polyakov loops for critical strings and superstrings at finite temperature, Nucl. Phys. B381, 201 (1992).

[25] J. Polchinski, String Theory II, Cambridge University Press (1998). 
[26] U.H. Danielsson, G. Ferretti, I.R. Klebanov, Creation of fundamental strings by crossing D-branes, Phys. Rev. Lett. 79, 1984 (1997); hep-th/9705084.

O. Bergman, M.R. Gaberdiel, G. Lifschytz, Branes, orientifolds and the creation of elementary strings, Nucl. Phys. B509, 194 (1998); hep-th/9705130. 